\title{
Effectiveness of percutaneus needle electrolysis and eccentric exercise in chronic patellar tendinopathy
}

\author{
Entrellardat Tortillol E. ${ }^{1}$ \\ ${ }^{1}$ Centro Medico Florida Portazgo, Alicante, Spain \\ Rev Fisioter Invasiva 2019;2:75.
}

\begin{abstract}
Keywords

- chronic tendinopathy

- patellar tendon

- percutaneous needle electrolysis

- eccentric exercise

Aims The aim of this study was to evaluate the effect of percutaneous needle electrolysis together with exercise with eccentric loading for the treatment of chronic patellar tendinopathy.

Material and Methods An experimental study was performed including eight subjects medically diagnosed with chronic patellar tendinopathy. The sample included subjects with a mean age of 40 years old (SD:10), a sample of 10 tendons was obtained with over four months' evolution. Functionality was assessed using the VISA-P scale, pain was evaluated using the Visual Analog Scale (VAS), the tendon structure was evaluated by analyzing of ultrasound images which evaluated the AP diameter, perimeter and echogenicity. All study subjects belonged to the same group and were applied the same treatment. This consisted of three sessions of percutaneous needle electrolysis, following the protocol defined by Valera \& Minaya with a dosage of 3:3:3, with $3 \mathrm{~mA}$ of intensity for 3 seconds and 3 current applications, together with a program of eccentric overloading which was developed in nine exercise sessions, which consisted of three series of 12 repetitions single-legged semi squats with maximum patient load on a decline board. A 48-hour window was left between the clinical session and sports practice with eccentric overloading.

Results In the initial assessment, a VISA-P score of 40.90 was obtained (SD:10.55), together with a VAS a score of 7.3 (SD:1.01), on the ultrasound exam all patients presented changes compatible with a degenerative process. After four weeks, the values of the VISA-P scale increased $35.8 \%$ at four weeks, VAS decreased $55.4 \%$ and slight changes were appreciated in ultrasound values in $42.4 \%$ of patients.

Conclusion Percutaneous needle electrolysis, together with eccentric overload exercise, represent a treatment which may be effective for most cases of chronic patellar tendinopathy.
\end{abstract}

